\title{
Neue Fragestellungen, Methoden und Ergebnisse der Permafrostforschung in der letzten Dekade
}

\author{
Michael Krautblatter ${ }^{1}$, Lutz Schirrmeister ${ }^{2}$, and Josefine Lenz ${ }^{2,3}$ \\ ${ }^{1}$ Fachgebiet Hangbewegungen, Technische Universität München, München, Deutschland \\ ${ }^{2}$ Alfred-Wegener-Institut Helmholtz-Zentrum für Polar- und Meeresforschung, Potsdam, Deutschland \\ ${ }^{3}$ Association of Polar Early Career Scientists, International Directorate, Potsdam, Deutschland \\ Correspondence: Michael Krautblatter (m.krautblatter@tum.de)
}

Published: 20 May 2021

\section{Wichtige Fragestellungen, Methoden und Ergebnisse der Permafrostforschung}

Permafrost ist rein thermisch dadurch definiert, dass Fels, Lockermaterial oder Boden für mindestens zwei aufeinanderfolgende Jahre unter $0^{\circ} \mathrm{C}$ bleiben, unabhängig vom Wassergehalt des Materials. Diese Definition fällt oft nicht mit dem tatsächlichen Gefrierpunkt zusammen, da z.B. Gesteinsdruck, gelöste Ionen im Porenwasser und die Krümmung von kleinen Porenräumen den Gefrierpunkt verändern. Auch unterhalb des Gleichgewichtsgefrierpunkts gibt es noch flüssiges Wasser, das erst beim Erreichen eines noch tieferen spontanen Gefrierpunkts friert. Permafrost ist im Gegensatz zu Gletschern eine meist versteckte Vereisung, die dadurch in Erscheinung tritt, dass sich das thermische, hydraulische, mechanische und biotische Verhalten der gefrorenen Felsen, Lockermaterialien oder des Bodens abrupt ändert. Während die Erforschung der Vergletscherung der Alpen und polarer Gebirge auf eine mehr als 150-jährige Geschichte zurück blickt, rückte der Permafrost, als verborgene Vereisung im Innern von Böden, Schuttmassen und Felsen in den polaren Gebieten erst 1909 mit Walery von Lozinski in den Fokus und wird in den Hochgebirgen erst seit den ausgehenden 70er Jahren verstärkt untersucht. Für die Veränderungen von Permafrost in polaren Gebieten und im Hochgebirge gibt bis auf wenige Ausnahmen erst seit den 1960er bzw. 1980er Jahren verlässliche Messreihen, was die Analyse der Auswirkungen des Klimawandels heute erheblich erschwert.

Die Quantifizierung und Modellbildung der Geowissenschaften hat auch die Permafrostforschung in der letzten Dekade erheblich verändert. Als übergeordnete neue Fragestellungen haben sich folgende Trends herausgestellt:
1. Thermische Modelle. Sowohl für den polaren als auch für den Hochgebirgspermafrost wurden großräumige thermische Modelle entwickelt, die Strahlungshaushalt, latente und sensible Wärmeflüsse berücksichtigen. Für zahlreiche lokale Einflussfaktoren wie Schnee- und Vegetationsbedeckung und komplexe 3D Topographie wurden und werden zahlreiche spezielle Modelle entwickelt.

2. Hydrologische Modelle. Es gibt einen umfangreichen Trend zur hydrologischen Modellbildung und auch Benchmarks an denen die Effektivität der hydrologischen Modelle getestet werden kann.

3. Mechanische Modelle. Sowohl im alpinen als auch im polaren Bereich wurden mechanische Modelle entwickelt die den Einfluss des gefrorenen und getauten Untergrundes zu quantifizieren versuchen.

4. Ökosystemare Modelle. Die Einwirkung der Permafrostdegradation auf Vegetation und polare/hochalpine Ökosysteme ist ein jüngst rasch expandierendes Forschungsthema.

5. Paläoumweltentwicklung und Geochronologie der Permafrostlandschaften. Neben der Weiterführung der klassischen kryolithologischen und paläontologischen Forschungen haben sich neue Methoden zur Untersuchung alter DNA und mikrobieller Prozesse in den Jahrzehntausende alten gefrorenen Ablagerungen entwickelt und etabliert. Diese ermöglichen wesentlich breitere Untersuchungen der vergangenen ökologischen Entwicklung vor allem in den polaren Gebieten. Weiterentwickelte geochronologische Methoden, vor al- 
lem der Lumineszenzdatierungen, ermöglichen exaktere und wesentlich weiter in die Vergangenheit reichende Altersbestimmungen.

6. Kohlenstoffkreislauf und-emissionen. Zahlreiche Publikationen des letzten Jahrzehnts befassen sich mit der Berechnung und der Charakterisierung der organischen Substanz, die in Permafrostablagerungen eingefroren ist und eine wichtige Treibhausgasquelle bei der heutigen und zukünftigen verstärkten Erwärmung der polaren Landschaften darstellt. Biomarker, ursprünglich in der Erdölforschung genutzt, haben sich als hervorragende Indikatoren für die Qualität der gefrorenen organischen Substanz erwiesen.

7. Geophysikalisches 4D Monitoring. Refraktionsseismik, elektrische Resistivitäts-/Impedanz-Tomographie, Bodenradar und weiter Methoden werden zunehmend eingesetzt um quantitative Parameter wie Eisgehalte, Wassergehalte und thermische Bedingungen abzuschätzen in 2D und 3D. Änderungen werden über wiederholtes Monitoring quantifiziert und können so invasionsfreie Einblicke in 4D Permafrost-Systementwicklung generieren.

8. Fernerkundliches Hochskalieren von Permafrosterscheinungen und deren Degradation. Fernerkundungsmethoden in engem Zusammenhang mit GIS-Methoden haben sich in den vergangenen Jahren für polaren und Hochgebirgsregionen rasant entwickelt. Zum einen haben sich die Techniken bei der Auswertung älterer Luft- und Satellitenbilder entschieden weiterentwickelt. Zum anderen werden neue Hochleistungssatelliten eingesetzt, die zum Teil auch speziell für die Permafrostforschung Daten erstellen. Die Kartierungen schwer zugänglicher Gebiete der Arktis unter verschiedensten Fragestellungen, die spektrale Untersuchungen permafrostbedingter Phänomene, sowie die Zeitreihenanalysen und Entwicklung automatisierter Auswertungsmethoden haben durch technische Entwicklung eine enorme Aufwertung erfahren.

9. Entwicklung polarer Küsten- und Flusssysteme. Die Berechnung von Abtragungsraten an Küsten und Flussufern und der Stoffeintrag in unterschiedliche aquatische Systeme bilden einen Schwerpunkt der polaren Küstenforschung. Zugleich wurden verstärkt Untersuchungen zur stofflichen Charakteristik des erodierten, transportierten und re-sedimentierten Materials vorgenommen. Dabei kommen neben Felduntersuchungen, Fernerkundungs- und GIS-Methoden, zunehmend auch moderne biogeochemische Methoden zum Einsatz.

10. Naturgefahren mit Permafrostdegradation. Die mit der Permafrostdegradation einhergehenden unmittelbaren Naturgefahren und -risiken rücken zunehmend in den Fokus der Forschung. Rasche Permafrostdegradation im
Wirkumfeld von Infrastruktur in polaren Gebieten führt zunehmend zu geotechnischen Problemen. In alpinen Gebieten und auf dem tibetischen Hochplateau werden Veränderungen der Permafrostsysteme zunehmend in die Planung von Infrastruktur einbezogen und entsprechende Mess- und Modellierungsverfahren entwickelt sowie geotechnische Maßnahmen entwickelt.

11. Interaktion Permafrost und Infrastruktur/Mensch. Die Ingenieurtechnische Modellierung des Einflusses von Permafrostdegradation auf Infrastrukturelemente ist in den Alpen Forschungsfront und in den polaren Permafrostgebieten etabliert; der interdisziplinäre Austausch ist regional noch unterschiedlich stark entwickelt. Die Verknüpfung natur- und sozialwissenschaftlicher Forschungsansätze im letzten Jahrzehnt beleuchtet zunehmend gesellschaftliche Anpassungsprozesse in polaren und alpinen Gebieten.

\section{Interview mit Prof. Dr. Michael Krautblatter and Dr. Lutz Schirrmeister}

Prof. Dr. Michael Krautblatter und Dr. Lutz Schirrmeister leiten seit 2011 gemeinsam den Arbeitskreis Permafrost. Warum ihnen besonders der Nachwuchs der Polarforschung am Herzen liegt und was die wichtige Fragen für ihren Arbeitskreis sind, erläutern Herr Krautblatter und Herr Schirrmeister im Interview.

APECS Germany: Kurz zu Ihrer Person und Ihrem beruflichen Werdegang. Wollten Sie schon immer Polarforscher werden? Wann und warum haben Sie sich für die Polarforschung entschieden?

M. Krautblatter: Wie zu den meisten Dingen im Leben komme ich auch zu den Polargebieten über mehrere Weichen. Vom Steinschlag im Wettersteingebirge in meiner Abschlussarbeit bin ich bei einem anschließenden dreimonatigen Forschungsaufenthalt in Oxford zur Verwitterung in kalten Klimaten gekommen. Von da hats mich dann auf eine Promotionsstelle zu Permafrost nach Bonn gezogen, die Felsstürze habe ich mitgenommen. Und auf einer Tagung des AK Permafrost habe ich dann Hughes Lantuit, Michael Fritz und Josefine Lenz getroffen, mit denen ich zwei wunderbare Sommer auf Herschel Island forschen durfte. Sie haben mich sozusagen angesteckt; ich war inzwischen auch noch in Sibirien und Spitzbergen und wir haben jetzt ein Projekt mit der DTU in Grönland, aber ich bin sozusagen Polarforscher auf dem zweiten Bildungsweg.

L. Schirrmeister: 1960 geboren, verheiratet, 6 Kinder, 1981-1989 in Greifswald Geologie studiert und promoviert, von 1989-1997 an der Humboldt-Universität zu Berlin (HUB), seit 1998 am AWI in Potsdam.

APECS Germany: Wollten Sie schon immer Polarforscher werden?

L. Schirrmeister: Nein, ich habe vorher an der HUB acht Jahre als Quartärgeologe in Brandenburg gearbeitet und mich 
davor mit lateritischer Verwitterung auf Sardinien, in Vietnam und Burundi beschäftigt.

APECS Germany: Wann und warum haben Sie sich für die Polarforschung (oder Permafrostforschung) entschieden?

L. Schirrmeister: Ich bin 1998 auf die Ausschreibung einer Stelle im BMBF Projekt „System Laptev Sea 2000“ im Teilprojekt „,Terrestrische Klimasignale in Permafrostablagerungen“ gestoßen. Die AWI-Forschungsstelle Potsdam begann damals im großen Umfang mit neuen Forschungen zu Permafrostprozessen in Nordostsibirien. Ich war auf der Suche nach neuer und interessanter Arbeit, da meine Beschäftigungsmöglichkeiten am Geographischen Institut der HUB ausgeschöpft waren.

APECS Germany: Was gefällt Ihnen an Ihren Aufgaben als AK Leiter ganz besonders?

L. Schirrmeister: Ich habe die Aufgaben als Ko-Sprecher des Arbeitskreises Permafrostforschung in der Deutschen Gesellschaft für Polarforschung 2008 übernommen. Es ist gut, dass diese kleine, aber recht aktive deutschsprachige Wissenschaftlergemeinschaft eine gemeinsame Plattform hat. Es gab seit dem Ende des letzten Jahrhunderts das Deutsche Nationalkomitee Permafrost, dass vor allem die internationalen Belange der Permafrostforscher Deutschlands vertrat.

Mit dem Arbeitskreis Permafrost in der DGP hat sich das Wirkungsfeld auf die deutschsprachigen Länder Deutschland, Österreich und Schweiz erweitert. Es wurden vor allem stärker junge Forscher (Studenten, Doktoranden und Postdocs) mit einbezogen. Zudem sind Forscher in Verantwortung aus verschiedenen Forschungseinrichtungen und Wissenschaftler im Ruhestand in die Arbeit des Arbeitskreises einbezogen. Diese Mischung und ein niederschwelliges Angebot für junge Kollegen bei den jährlichen Treffen liegen mir besonders am Herzen.

APECS Germany: Hat sich dadurch Ihre Sicht auf etwas geändert?

L. Schirrmeister: Es geht zum einen international darum hochrangige Projekte und Publikationen $\mathrm{zu}$ realisieren. Es geht zum anderen aber auch darum so eine kleine Gemeinschaft, wie es die Permafrostforschung nun einmal ist, zusammenzuhalten und international sichtbar zu machen. Dafür ist der AK Permafrost ein sehr gutes Podium.

APECS Germany: Warum setzen Sie sich für das Thema Ihres AK ein? Was macht dieses Forschungsfeld für Sie so besonders, dass Sie dafür so viel Zeit und Einsatz bringen?

M. Krautblatter: Ich mag Themen die ganz unterschiedliche Forscher zusammen bringen, ich finde da ist die Lernkurve am steilsten. Und der Permafrost ist ein solches Thema bei dem man, ehe man sich versieht, auf einer Insel mit Mikrobiolog*Innen, Botaniker*Innen, Geomorpho$\log$ *Innen, Geophysiker*Innen für drei Woche haust und das meiste außerhalb der eigenen Domäne lernt. Ich finde diesen Austausch beim Forschen und auf den Tagungen großartig und ich möchte nicht mehr darauf verzichten.
APECS Germany: Wenn Sie in die Zukunft schauen, was würden Sie gerne im Feld Ihres Arbeitskreises in fünf Jahren herausgefunden haben? Was ist in Ihren Augen die dringendste Frage im Feld Ihres Arbeitskreises?

M. Krautblatter: Die Welt der Permafrostsysteme befindet sich in einem unheimlich schnellen Wandel den wir gerade erst anfangen zu verstehen in seiner Tragweite und wir befinden uns am Anfang einer steilen Lernkurve was die Auswirkungen betrifft. Wir haben hier einen gesellschaftlichen Auftrag erster Güte, nämlich die Personen, die es betrifft und die Gesellschaft vorzubereiten. In den Alpen ist das heute schon Teil unserer Aufgabe und wir sprechen und planen mit den Hüttenwarten, dem Alpenverein, den Seilbahnbesitzern und Bürgermeistern. Die Aufgabe, der wir uns stellen müssen wird nicht in fünf Jahren beendet sein aber wir müssen jetzt damit anfangen.

L. Schirrmeister: Ob der Permafrost in den polaren Gebiete und den Hochgebirgen längerfristig eine Chance hat erhalten zu bleiben. Was muss dafür getan werden?

APECS Germany: Was ist in Ihren Augen die dringendste Frage im Feld Ihres Arbeitskreises?

L. Schirrmeister: Welche Alternativen gibt es für die Bevölkerung in den Permafrostregionen, wenn die Permafrostdegardation weiter voranschreitet?

APECS Germany: Was war Ihre einprägsamste (lustig/beängstigend/seltsam) Erfahrung in der Polarforschung?

M. Krautblatter: Die Wochen auf Herschel Island werde ich nie vergessen. In den Alpen arbeiten wir oft hochgetaktet und es ist jede halbe Stunde ausgeplant bis zum Abstieg oder der letzten Seilbahnfahrt. Die Abgeschiedenheit auf Herschel von äußerer Information, die täglich weitergehenden Unterhaltungen mit den Teilnehmern, die Sauna neben den driftenden Eisschollen im Pauline Cove und der langsame entschleunigte Takt der sich im Polartag sich neben der Arbeit einstellt.

Interessenkonflikt. Die Autor*innen erklären, dass kein Interessenkonflikt besteht.

Begutachtung. This paper was edited by Donovan Dennis. 